\section{Entire functions of restricted hyper-order sharing a set of two small functions IM with their linear c-shift operators}

Entire functions of restricted hyper-order

Abhijit Banerjee and Arpita Roy

Department of Mathematics, University of Kalyani, Kalyani, India

\begin{abstract}
Purpose - The paper aims to build the relationship between an entire function of restricted hyper-order with its linear c-shift operator.

Design/methodology/approach - Standard methodology for papers in difference and shift operators and value distribution theory have been used.

Findings - The relation between an entire function of restricted hyper-order with its linear c-shift operator was found under the periphery of sharing a set of two small functions IM (ignoring multiplicities) when exponent of convergence of zeros is strictly less than its order. This research work is an improvement and extension of two previous papers.
\end{abstract}

Originality/value - This is an original research work.

Keywords Entire functions, Exponent of convergence, Hyper-order, Linear c-shift operator, Shared set

Paper type Research paper

\section{Introduction}

By a meromorphic function $f$, we always mean that it is defined on $\mathbb{C}$. For such a meromorphic function, we recall some basic terminologies of value distribution theory such as the Nevanlinna characteristic function $T(r, f)$, the proximity function $m(r, f)$ and the counting function (reduced counting function) of $a$-points of $f N\left(r, \frac{1}{f-a}\right)=N(r, a ; f)\left(\bar{N}\left(r, \frac{1}{f-a}\right)=\right.$ $\bar{N}(r, a ; f))$. For $a=\infty$, we use $N(r, f)=N(r, \infty ; f)(\bar{N}(r, f)=\bar{N}(r, \infty ; f))$ to denote counting (reduced counting) function of poles of $f$ (see [1]). With the help of the standard notations, we also would like to recall the following useful terms, namely exponent of convergence of zeros, order and hyper-order of $f$ respectively defined as follows:

$$
\lambda(f)=\limsup _{r \rightarrow \infty} \frac{\log N\left(r, \frac{1}{f}\right)}{\log r}, \rho(f)=\limsup _{r \rightarrow \infty} \frac{\log T(r, f)}{\log r} \text { and } \rho_{2}(f)=\limsup _{r \rightarrow \infty} \frac{\log \log T(r, f)}{\log r} .
$$

\section{JEL Classification - 39A70, 30D35}

(C) Abhijit Banerjee and Arpita Roy. Published in Arab Journal of Mathematical Sciences. Published by Emerald Publishing Limited. This article is published under the Creative Commons Attribution (CC BY 4.0) licence. Anyone may reproduce, distribute, translate and create derivative works of this article (for both commercial and non-commercial purposes), subject to full attribution to the original publication and authors. The full terms of this licence may be seen at http://creativecommons.org/licences/by/4.0/ legalcode

The authors wish to thank the referee for a careful reading and valuable suggestions toward the improvement of the paper. The first author is thankful to DST-PURSE -II Programme for financial assistance. The second author is thankful to the Council of Scientific and Industrial Research (India) for their financial support under File No: 09/106 (0188)/2019- EMR-I.
Received 26 October 2020

Revised 26 May 2021

Accepted 1 July 2021

\section{.}


AJMS 28,2

Usually, $S(r, f)$ denotes any quantity satisfying $S(r, f)=o(T(r, f))$ for all $r$ outside of a possible exceptional set of finite linear measure. We denote by $S(f)$ the set of all meromorphic functions $a(z)$ such that $T(r, a(z))=S(r, f)$ and $a(z)$ is called small function compared to $f(z)$. Let $a(z) \in S$ and $S$ be a subset of $S(f) \cup\{\infty\}$ and $E_{f}(S)=\cup_{a(z) \in S}\{z: f(z)-a(z)=0\}$, where each zero is counted according to its multiplicity. If we do not count the multiplicity, then the set $\cup_{a(z) \in S}\{z$ : $f(z)-a(z)=0\}$ is denoted by $\bar{E}_{f}(S)$.

If $E_{f}(S)=E_{g}(S)\left(\bar{E}_{f}(S)=\bar{E}_{g}(S)\right)$ we say that $\mathrm{f}$ and $\mathrm{g}$ share the set $\mathrm{S} \mathrm{CM}$ or counting multiplicities (IM or ignoring multiplicities).

For a nonzero complex constant $c$, the shift operator of $f(z)$ is denoted by $f(z+c)$. The terms $\Delta_{c} f$ and $\Delta_{c}^{k} f$ will be used to denote the difference and $k$-th order difference operators of $f(z)$, defined respectively as

$$
\Delta_{c} f(z)=f(z+c)-f(z), \Delta_{c}^{k} f(z)=\Delta_{c}\left(\Delta_{c}^{k-1} f(z)\right), \quad k \in \mathbb{N}, \quad k \geq 2 .
$$

We introduce the more generalized linear $c$-shift operator $L_{c} f$ by

$$
L_{c} f=L_{c}(f)(z)=\sum_{j=0}^{k} a_{j} f(z+j c)(\not \equiv 0),
$$

where $a_{j} \in \mathbb{C}$ for $j=0,1,2, \ldots, k$ with $a_{k} \neq 0(k \geq 1)$.

The uniqueness problem of entire functions sharing set with their derivatives, shifts, different types of difference operators has been developed as an interesting direction of research in the realm of value distribution theory. In 1999, Li-Yang [2] made a pioneer work by considering the relation between an entire function and its derivative sharing a set with two elements. Following their footsteps, in 2005, Li [3] investigated the same type of problem for linear differential operator. Four years later, Liu [4] exhibited a similar result for an entire function $f$ and its shift sharing a set with two small functions.

Let us start the discussion with another result of Liu [4] concerning difference operator.

Theorem A. [4] Let fbe a transcendental entire function of finite order, and let a be a nonzero finite constant. If $f$ and $\Delta_{c} f$ share the set $\{a,-a\} C M$, then $\Delta_{c} f=f$.

After that Liu [4] posed a significant question:

Question 1.1. What happens if $\{a,-a\}$ is replaced by $\{a(z), b(z)\}$ in the above theorem, where $a(z), b(z) \in S(f)$ are nonvanishing periodic entire functions with period $c$ ?

Being motivated by this question, Li [5] investigated the following theorem in a different direction that evolved as a new trend. Actually, Li [5] first diverted the attention of the research germinated from Question 1.1, in terms of relation between exponent of convergence of zero and order. We recall the theorem by $\mathrm{Li}$ [5].

Theorem B. [5] Let $f$ be a nonconstant entire function such that $\lambda(f)<\rho(f)<\infty, \rho(f) \neq 1, a, b$ be respectively two distinct entire functions such that $\rho(a)<\rho(f)$ and $\rho(b)<\rho(f)$. Iff and $\Delta_{c} f$ share the set $\{a, b\} C M$, then $\Delta_{c} f=$ f for all $z \in \mathbb{C}$.

By an example we can show that the restriction $\rho(f) \neq 1$ in Theorem B can be removed.

Example 1.1. Let $f(z)=e^{\frac{z \log 2}{c}}$. Then obviously $\Delta_{c} f=f$. Clearly $f$ and $\Delta_{c} f$ share the set $\{a, b\}$ $C M$ for two distinct entire functions $a$, $b$ respectively such that $\rho(a)<\rho(f)$ and $\rho(b)<\rho(f)$ and also $0=\lambda(f)<\rho(f)=1$.

After publication of Li's [5] result, there was a long gap in research in this direction. Recently in 2019, concerning finite-order entire function, Qi-Wang-Gu [6] removed the restriction $\rho(f) \neq 1$ in Theorem B. Not only that, they also ensured the particular form of $f$ in the following manner: 
Theorem C. [6] Let $f$ be a nonconstant entire function with $\lambda(f)<\rho(f)<\infty$, let $a$, $b$ be respectively two distinct entire functions such that $\rho(a)<\rho(f), \rho(b)<\rho(f)$. If f and $\Delta_{c} f$ share the set $\{a, b\} C M$, then $f(z)=A e^{\mu z}$, where $A, \mu$ are two nonzero constants satisfying $e^{\mu c}=2$. Furthermore, $\Delta_{c} f=f$.

Entire functions of restricted hyper-order

\section{Main results}

In our paper, we have extended and improved Theorem $\mathrm{C}$ in the following three directions:

(1) We replace the difference operator by its linear $c$-shift operator to accommodate a larger class of operators, namely $L_{c} f$ that includes difference operator.

(2) We consider an entire function of $\rho_{2}(f)<1$ instead of considering the same of finite order.

(3) We relax the nature of the shared set $\{a, b\}$ from CM to IM.

Thus, the following assertion extends and improves Theorem $\mathrm{C}$ in the way described above, and in fact it represents our main result in this paper.

Theorem 2.1. Let fbe a nonconstant entire function such that $\lambda(f)<\rho(f)$ with $\rho_{2}(f)<1$ and let $a, b$ be two distinct entire functions such that $\rho(a)<\rho(f)$ and $\rho(b)<\rho(f)$. Let $f$ and $L_{c} f(\not \equiv 0)$ share the set $\{a, b\} I M$, then $L_{c} f=f$. In addition, if $b=-a$, then $L_{c} f=-f$. In both cases $f$ takes the form $f(z)=A h(z) e^{\mu z}$, where $A$ is a nonzero constant, $h(z)$ is a polynomial and $\mu$ is a nonzero constant satisfying $\sum_{j=0}^{k} a_{j} e^{\mu c j}=1$ and -1 respectively. Furthermore,

(1) when $L_{c} f=f$, then one of the following can occur:

- If $a_{0}=1$, then $k \geq 2$ and $\operatorname{deg}(h) \leq(k-2)$;

- If $a_{0} \neq 1$, then $\operatorname{deg}(h) \leq(k-1)$.

(2) When $L_{c} f=-f$, then one of the following occur:

- if $a_{0}=-1$, then $k \geq 2$ and $\operatorname{deg}(h) \leq(k-2)$;

- if $a_{0} \neq-1$, then $\operatorname{deg}(h) \leq(k-1)$.

Remark 2.1. In the above theorem, if we choose $L_{c} f=\Delta_{c} f$, then $k=1, a_{1}=1$ and $a_{0}=-1$. Therefore conclusion (2) that means $\Delta_{c} f=-$ fis not possible. Thus from conclusion (1) we only have the form of the function as $f(z)=A e^{\mu z}$, where $A$ and $\mu$ are nonzero constants satisfying $e^{\mu c}=2$ and also $\Delta_{c} f=$ f holds.

The following examples will successively show that in the above theorem, respectively for the cases $k=1, k=2$ and $k=3$, all possible forms of the function exist.

First we consider the case $k=1$.

Example 2.1. Let $f=A e^{\mu z}, A \neq 0$. Choosing coefficients of $L_{c} f$ for $k=1$ as $a_{1}=\frac{1-a_{0}}{e^{\mu c}}$ and $a_{0} \neq 1$ we have $L_{c} f=f$ and $\sum_{j=0}^{1} a_{j} e^{\mu c j}=1$. Next, choosing coefficients as $a_{1}=\frac{-1-a_{0}}{e^{\mu c}}$ and $a_{0} \neq-1$ we see that $L_{c} f=-f$ and $\sum_{j=0}^{1} a_{j} e^{\mu c j}=-1$.

Next we shall show that for $k=2$, the forms of the function can be obtained.

Example 2.2. Let $f=(A z+B) e^{\mu z}, A \neq 0$. Choosing coefficients of $L_{c} f$ for $k=2$ as $a_{2}=\frac{-\left(1-a_{0}\right)}{e^{2 \mu c}}, a_{1}=\frac{2\left(1-a_{0}\right)}{e^{\mu c}}$, one can easily check that $L_{c} f=f$ and $\sum_{j=0}^{2} a_{j} e^{\mu c j}=1$. On the other 
AJMS

28,2

hand, choosing coefficients as $a_{2}=\frac{1+a_{0}}{e^{2 \mu c}}, a_{1}=\frac{-2\left(1+a_{0}\right)}{e^{\mu c}}$, we easily can obtain $L_{c} f=-f$ and $\sum_{j=0}^{2} a_{j} e^{\mu c j}=-1$.

Example 2.3. Consider the function fin Example 2.1. Choose coefficients of $L_{c} f$ for $k=2$ as $a_{2}=\frac{-4}{e^{2 \mu \omega}} a_{1}=\frac{3}{e^{\mu \omega}}, a_{0}=2$, then we have $L_{c} f=$ fand $\sum_{j=0}^{2} a_{j} e^{\mu c j}=1$. Next, choosing coefficients as $a_{2}=\frac{-6}{e^{2 \mu}} a_{1}=\frac{3}{e^{\mu \omega}}, a_{0}=2$, we see that $L_{c} f=-f$ and $\sum_{j=0}^{2} a_{j} e^{\mu c j}=-1$.

For $k=3$, all possible forms of the function are shown below.

Example 2.4. Let $f=\left(A z^{2}+B z+C\right) e^{\mu z}, A \neq 0$. Choosing coefficients of $L_{c} f$ for $k=3$ as $a_{3}=\frac{1-a_{0}}{e^{3 \mu c}}, a_{2}=\frac{-3\left(1-a_{0}\right)}{e^{2 \mu c}}, a_{1}=\frac{3\left(1-a_{0}\right)}{e^{\mu c}}$, one can easily check that $L_{c} f=f$ and $\sum_{j=0}^{3} a_{j} e^{\mu c j}=1$. Also, choosing coefficients as $a_{3}=\frac{-1-a_{0}}{e^{3 \mu c}}, a_{2}=\frac{-3\left(-1-a_{0}\right)}{e^{2 \mu c}}, a_{1}=\frac{3\left(-1-a_{0}\right)}{e^{\mu c}}$, one can easily check that $L_{c} f=-f$ and $\sum_{j=0}^{3} a_{j} e^{\mu c j}=-1$.

Example 2.5. Consider the function fas in Example 2.2 and choosing the coefficients of $L_{c} f$ as $a_{3}=\frac{5}{e^{3 \mu \omega}} a_{2}=\frac{-9}{e^{2 \mu \omega}} a_{1}=\frac{3}{e^{\mu \omega}}, a_{0}=2$, we can have $L_{c} f=f$ and $\sum_{j=0}^{3} a_{j} e^{\mu c j}=1$. On the other hand, choosing coefficients as $a_{3}=\frac{9}{e^{3 \mu \omega}}, a_{2}=\frac{-15}{e^{2 \mu c}}, a_{1}=\frac{3}{e^{\mu(\omega)}}, a_{0}=2$, we can get $L_{c} f=-f$ and $\sum_{j=0}^{3} a_{j} e^{\mu c j}=-1$.

Example 2.6. Consider the function fin Example 2.1. Choosing coefficients of $L_{c} f$ for $k=3$ as $a_{3}=\frac{-2}{e^{3 \mu \nu}} a_{2}=\frac{4}{e^{2 \mu \omega}} a_{1}=\frac{-3}{e^{\mu \omega}}, a_{0}=2$, clearly $L_{c} f=f$ and $\sum_{j=0}^{3} a_{j} e^{\mu c j}=1$. On the other hand, choosing coefficients as $a_{3}=\frac{-4}{e^{3 \mu \omega}}, a_{2}=\frac{4}{e^{2 \mu \omega}} \quad a_{1}=\frac{-3}{e^{\mu \omega}}, a_{0}=2$, we easily get $L_{c} f=-f$ and $\sum_{j=0}^{3} a_{j} e^{\mu c j}=-1$.

Similar examples can be constructed for the case $k \geq 4$ also.

Remark 2.2. In Examples 2.4, 2.5, let us take $L_{c} f=\Delta_{c}^{3} f$. Choosing $e^{\mu c}=2$ we see that though $\sum_{j=0}^{3} a_{j} e^{\mu c j}=\left(e^{\mu c}-1\right)^{3}=1$ but $\Delta_{c}^{3} f \neq f$. In a similar manner, for the function in Examples 2.4, 2.5, choosing $e^{\mu c}=\frac{3+\sqrt{3} i}{2}$ we get $\sum_{j=0}^{3} a_{j} e^{\mu c j}=\left(e^{\mu c}-1\right)^{3}=-1$ but $\Delta_{c}^{3} f \neq-f$. But in Example 2.6, choosing $e^{\mu c}$ such that $\sum_{j=0}^{3} a_{j} e^{\mu c j}=1$ or -1 , we automatically have the respective conclusions $\Delta_{c}^{3} f=$ for $\Delta_{c}^{3} f=-$ f. From this observation naturally one can infer that the case $L_{c} f=\Delta_{c}^{k} f$ needs special attention. In fact, we can conjecture that the degree of $h$ could be zero that means $h$ will be a nonzero constant.

In this respect, in Theorem 2.1 replacing $L_{c} f$ by $\Delta_{c}^{k} f$ we can get the next corollary.

Corollary 2.1. Under the same assumptions of Theorem 2.1 for the operator $\Delta_{c}^{k} f$ we have $\Delta_{c}^{k} f=f$. In addition, if $b=-a$ and $k \geq 2$, then $\Delta_{c}^{k} f=-f$. In both cases $f$ takes the form $f(z)=B e^{\mu z}$, where $B$ and $\mu$ are nonzero constants and $\mu$ satisfies $\sum_{j=0}^{k}(-1)^{k-j}\left(\begin{array}{l}k \\ j\end{array}\right) e^{\mu c j}=1$ and -1 respectively.

Next we provide two examples to show that $\rho_{2}<1$ is sharp.

Example 2.7. Let $f=e^{\frac{\pi i z}{c}} e^{e^{\frac{2 \pi i z}{c}}}$. Here $\lambda(f)<\rho(f)$ and $\rho_{2}(f)=1$. For a suitable choices of coefficients one can obtain $L_{c} f=-e^{\frac{\pi i z}{c}} e^{\frac{2 \pi i z}{c}}$. For example, for even integer $k$, choose $a_{k}+\cdots+a_{2}+a_{0}=0$ and $a_{k-1}+\cdots+a_{3}+a_{1}=1$. Clearly $f$ and $L_{c} f$ share the set $\{a,-a\} C M$, where $a$ is an entive function such that $\rho(a)<\rho(f)$. Though $L_{c} f=-f$, the form of $f$ does not satisfy the conclusion of our theorem. 
Example 2.8. Let $f=e^{\frac{\pi i z}{e}}$. Here $\lambda(f)<\rho(f)$ and $\rho_{2}(f)=1$. For a suitable choices of coefficients, one can easily obtain $L_{c} f=e^{-e^{\frac{\pi i z}{c}}}$. For example, for even integer $k$, choose $a_{k}+\cdots+a_{2}+a_{0}=0$ and $a_{k-1}+\cdots+a_{3}+a_{1}=1$. Clearly $f$ and $L_{c} f$ share the set $\{\sqrt{a}+\sqrt{b}, \sqrt{a}-\sqrt{b}\} C M$, where $a, b$ are two complex constants such that $a-b=1$. Then neither $L_{c} f= \pm$ fnor the form of $f$ satisfies the conclusion of our theorem.

Entire functions of restricted hyper-order

\section{Preparatory lemmas}

In this section, some useful lemmas are quoted from references [1, 7, 9-12], which will be needed in the sequel.

Lemma 3.1. [9] Let $T:[0,+\infty) \rightarrow[0,+\infty)$ be a nondecreasing continuous function, and let $s \in(0,+\infty)$. If the hyper-order of $T$ is strictly less than 1 , that is,

$$
\limsup _{r \rightarrow \infty} \frac{\log \log T(r)}{\log r}=\rho_{2}<1,
$$

and $\delta \in\left(0,1-\rho_{2}\right)$, then

$$
T(r+s)=T(r)+o\left(\frac{T(r)}{r^{\delta}}\right)
$$

where $r$ runs to infinity outside of a set of finite logarithmic measures.

Lemma 3.2. [9] Let $f(z)$ be a meromorphic function of $\rho_{2}(f)<1$ and $c \in \mathbb{C} \backslash\{0\}$. Then

$$
m\left(r, \frac{f(z+c)}{f(z)}\right)=o\left(\frac{T(r, f)}{r^{1-\rho_{2}-\epsilon}}\right) .
$$

Using the above two basic lemmas due to [9], we have the next lemma.

Lemma 3.3. Let $f(z)$ be a meromorphic function of $\rho_{2}(f)<1$ and $c \in \mathbb{C} \backslash\{0\}$. Then for any $\epsilon>0$,

$$
m\left(r, \frac{f(z+i c)}{f(z+j c)}\right)=o\left(\frac{T(r, f)}{r^{1-\rho_{2}-\epsilon}}\right) .
$$

Using Lemma 3.1, by a simple alteration of the result for finite-order meromorphic functions in [8], one can have the following lemma.

Lemma 3.4. Let $f(z)$ be a meromorphic function of $\rho_{2}(f)<1$, then we have

$$
N(r, f(z+c))=N(r, f)+S(r, f)
$$

and

$$
T(r, f(z+c))=T(r, f)+S(r, f) .
$$

Lemma 3.5. [7] Let $f$ be a transcendental meromorphic function in the plane of order less than 1. Let $h>0$. Then there exists an $\epsilon$-set $E$ such that

uniformly in c for $|c| \leq h$.

$$
\frac{g(z+c)}{g(z)} \rightarrow 1 \text {, when } z \rightarrow \infty \text { in } \mathbb{C} \backslash E,
$$


AJMS 28,2

222

Lemma 3.6. $[1,10]$ Let $f(z)$ be a transcendental meromorphic solution of equation

$$
f^{n} A(z, f)=B(z, f)
$$

where $A(z, f), B(z, f)$ are polynomials in $f$ and its derivatives with meromorphic coefficients, say $\left\{a_{\lambda}: \lambda \in I\right\}$, such that $m\left(r, a_{\lambda}\right)=S(r, f)$ for all $\lambda \in I$. If the total degree of $B(z, f)$ is $\leq n$, then

$$
m(r, A(z, f))=S(r, f)
$$

Lemma 3.7. (see [12], Theorem 1.51) Suppose that $f_{i}(z)(i=1,2, \ldots, n)$ and $g_{i}(z)(i=1,2, \ldots$, $n)(n \geq 2)$ are entire functions satisfying

(1) $\sum_{i=1}^{n} f_{i}(z) e^{g_{i}(z)} \equiv 0$,

(2) $g_{j}(z)-g_{k}(z)$ are not constants for $1 \leq j<k<n$,

(3) for $1 \leq i \leq n, 1 \leq k<l \leq n$,

$$
T\left(r, f_{i}\right)=o\left\{T\left(r, e^{g_{k}-g_{l}}\right)\right\} \quad(r \rightarrow \infty, r \notin E) .
$$

Then $f_{i}(z) \equiv 0(i=1,2, \ldots, n)$.

Now we recall the following lemma due to Lu-Lu-Li-Xu (see [11], Corollary 3.2).

Lemma 3.8. [11] Let $g(\not \equiv)$ be a nonconstant meromorphic solution of the linear difference equation

$$
\sum_{i=0}^{k} b_{i} g(z+i c)=R(z),
$$

where $R(z)$ is a polynomial and $b_{i}^{\prime} s$ for $i=0,1, \ldots k$ are complex constants with $b_{k} b_{0} \neq 0$, $c \in \mathbb{C} \backslash\{0\}$ and $k \in \mathbb{N}$. Then either $\rho(g) \geq 1$ or $g$ is a polynomial. In particular if $b_{k} \neq \pm b_{0}$, then $\rho(g) \geq 1$.

\section{Proof of the main theorem}

Proof of Theorem 2.1. According to our assumption $\lambda(f)<\rho(f)$ and by Hadamard factorization theorem, let us assume that $f(z)=h(z) e^{\eta(z)}$, where $h(z)(\not \equiv 0)$ is an entire function and $\eta(z)$ is a nonconstant entire function satisfying

$$
\lambda(f)=\rho(h)<\rho(f), \quad \rho\left(e^{\eta}\right)=\rho(f), \quad \rho(\eta)=\rho_{2}\left(e^{\eta}\right)=\rho_{2}(f)<1 .
$$

Therefore $T(r, h)=S(r, f)$ and $S\left(r, e^{\eta}\right)=S(r, f)=S(r)$. Here,

$$
T(r, f) \leq T(r, h)+T\left(r, e^{\eta}\right) \leq T\left(r, e^{\eta}\right)+S(r) .
$$

Let $q(z)=\frac{L_{c} f}{e^{\eta}}$. Clearly $q \not \equiv 0$. Then placing $f(z)=h(z) e^{\eta(z)}$, in view of Lemma 3.4, we can deduce that 


$$
\begin{aligned}
T(r, q)=m(r, q)=m\left(r, \frac{L_{c}\left(h(z) e^{\eta(z)}\right)}{e^{\eta(z)}}\right) & =m\left(r, \sum_{j=0}^{k} a_{j} h(z+j c) e^{\eta(z+j c)-\eta(z)}\right) \\
& \leq \sum_{j=0}^{k} m\left(r, \frac{e^{\eta(z+j c)}}{e^{\eta(z)}}\right)+S(r) \\
& =S\left(r, e^{\eta}\right)=S(r) .
\end{aligned}
$$

Since $q$ and $h$ are not equivalent to zero, one can easily write

$$
\frac{\left(q e^{\eta}-a\right)\left(q e^{\eta}-b\right)}{\left(h e^{\eta}-a\right)\left(h e^{\eta}-b\right)}=\frac{q^{2}\left(e^{\eta}-\frac{a}{q}\right)\left(e^{\eta}-\frac{b}{q}\right)}{h^{2}\left(e^{\eta}-\frac{a}{h}\right)\left(e^{\eta}-\frac{b}{h}\right)} .
$$

Applying the Second Fundamental Theorem for small functions [1] on $e^{\eta}$ and then applying the First Fundamental Theorem [1] on $e^{\eta}-\omega$, we can obtain that

$$
T\left(r, e^{\eta}\right)=\bar{N}\left(r, \frac{1}{e^{\eta}-\omega}\right)+S(r)
$$

where $\omega(\not \equiv 0)$ is a small function of $e^{\eta}$.

Here $a \not \equiv b$. Without loss of generality, let us assume that $a \not \equiv 0$. Let $z_{0}$ be a zero of $e^{\eta}-\frac{a}{h}$ but $q\left(z_{0}\right) \neq 0$. Since $f$ and $L_{c} f$ that means $h e^{\eta}$ and $q e^{\eta}$ share the set $\{a, b\}$ IM, so in view of (4.2), $z_{0}$ is a zero of $e^{\eta}-\frac{a}{q}$ or $e^{\eta}-\frac{b}{q}$. Let us denote by $\bar{N}\left(r, 0 ; e^{\eta}-\frac{a}{h}, e^{\eta}-\frac{a}{q}\right)$ the reduced counting function of those common zeros of $e^{\eta}-\frac{a}{h}$ and $e^{\eta}-\frac{a}{q}$, which are not zeros of $q$. Similarly, we denote by $\bar{N}\left(r, 0 ; e^{\eta}-\frac{a}{h}, e^{\eta}-\frac{b}{q}\right)$ the reduced counting function of those common zeros of $e^{\eta}-\frac{a}{h}$ and $e^{\eta}-\frac{b}{q}$, which are not zeros of $q$. Therefore from (4.3) we have,

$$
\begin{aligned}
T\left(r, e^{\eta}\right) & =\bar{N}\left(r, \frac{1}{e^{\eta}-\frac{a}{h}}\right)+S(r) \\
& =\bar{N}\left(r, 0 ; e^{\eta}-\frac{a}{h}, e^{\eta}-\frac{a}{q}\right)+\bar{N}\left(r, 0 ; e^{\eta}-\frac{a}{h}, e^{\eta}-\frac{b}{q}\right)+S(r),
\end{aligned}
$$

which shows that either $\bar{N}\left(r, 0 ; e^{\eta}-\frac{a}{h}, e^{\eta}-\frac{a}{q}\right) \neq S(r)$ or $\bar{N}\left(r, 0 ; e^{\eta}-\frac{a}{h}, e^{\eta}-\frac{b}{q}\right) \neq S(r)$. Otherwise $T\left(r, e^{\eta}\right)=S(r)$. This is not possible because in view of (4.1), we can draw a contradiction. Now, we consider two cases: Case 1. Suppose $\bar{N}\left(r, 0 ; e^{\eta}-\frac{a}{h}, e^{\eta}-\frac{a}{q}\right) \neq S(r)$. For sake of convenience, we resolve the case
step by step.

Step 1. In this step we will show that $L_{c} f \equiv f$.

Let $z_{1}$ is a zero of $e^{\eta}-\frac{a}{h}$ and $e^{\eta}-\frac{a}{q}$. It is obvious that $z_{1}$ is a zero of $\frac{a}{h}-\frac{a}{q}$. If $\frac{a}{h}-\frac{a}{q} \not \equiv 0$, then

$$
\bar{N}\left(r, 0 ; e^{\eta}-\frac{a}{h}, e^{\eta}-\frac{a}{q}\right) \leq \bar{N}\left(r, \frac{1}{\frac{a}{h}-\frac{a}{q}}\right)=S(r),
$$

which is a contradiction. Therefore $h=q$ that implies $L_{c} f=f$. 
Step 2. In this step we show that $\eta(z)$ is a polynomial.

28,2

Expanding $L_{c} f=f$ we can write

$$
\sum_{j=0}^{k} a_{j} h(z+j c) e^{\eta(z+j c)}=h(z) e^{\eta(z)} .
$$

Choosing $b_{j}=a_{j} h(z+j c)$ for $j=1,2, \ldots, k$ and $b_{0}=\left(a_{0}-1\right) h(z)$ we get $\sum_{j=0}^{k} b_{j} e^{\eta(z+j c)}=0$. Clearly $\rho\left(b_{l}\right)=\rho(h(z+l c))=\rho(h)<\rho(f)$ for all $l=0,1, \ldots, k$. So, $b_{l}^{\prime} s$ are finite-order entire functions. We claim that $\rho\left(e^{\eta(z+i c)-\eta(z+j c)}\right)<\infty$, for at least one pair of $i, j$; such that $0 \leq i<j \leq k$. On the contrary, let us suppose, for all $0 \leq i<j \leq k, \rho\left(e^{\eta(z+i c)-\eta(z+j c)}\right)=\infty$. Then $T\left(r, b_{l}\right)=o\left\{T\left(r, e^{\eta(z+j c)-\eta(z+i c)}\right)\right\}$, for $0 \leq l \leq k$ and $0 \leq i<j \leq k$. Hence by Lemma $3.7, b_{l} \equiv 0$ for all $l=0,1, \ldots, k$, which is not possible. Thereby $\rho\left(e^{\eta(z+i c)-\eta(z+j c)}\right)<\infty$ implies that $\eta(z+i c)-$ $\eta(z+j c)$ is a polynomial. Let the degree of $\eta(z+i c)-\eta(z+j c)$ be $m$. So, $\eta^{(m+1)}(z+i c)-$ $\eta^{(m+1)}(z+j c)=0$ that means $\eta^{(m+1)}(z+i c)$ is periodic entire function of period $(j-i) c$. If $\eta^{(m+1)}(z+i c)$ is nonconstant, then obviously $\rho\left(\eta^{(m+1)}(z+i c)\right) \geq 1$, which yields $\rho(\eta)=\rho(\eta(z+i c))=\rho\left(\eta^{(m+1)}(z+i c)\right) \geq 1$. But $\rho(\eta)<1$, a contradiction. Hence $\eta^{(m+1)}(z+i c)$ is a constant and so $\eta^{(m+1)}(z)$ is constant, which implies $\eta(z)$ is polynomial.

Step 3. In this step we wish to show that the degree of $\eta(z)$ is 1 .

On the contrary, suppose the $\operatorname{deg}(\eta(z))=n($ say $) \geq 2$. Then for $j=1,2, \ldots, k$,

$$
e^{\eta(z+j c)-\eta(z)}=e^{j c n c_{n} z^{n-1}+Q_{j}(z)},
$$

where $Q_{j}(z)$ is a $(n-2)$-th degree polynomial and $c_{n}$ is the leading coefficient of $\eta(z)$. Let $g=e^{c n n_{n} z^{n-1}}$. So, for $j=1,2, \ldots, k, e^{\eta(z+j c)-\eta(z)}=g^{j} e^{Q_{j}(z)}$. Clearly $T\left(r, e^{Q_{j}-Q_{k}}\right)=S(r, g)$ for all $j=1,2, \ldots, k-1$ and $T\left(r, e^{-Q_{k}}\right)=S(r, g)$. Here we will draw a contradiction by deducing $T(r, g)=S(r, g)$. Rewriting (4.4) we have

$$
\sum_{j=1}^{k} a_{j} \frac{h(z+j c)}{h(z)} e^{Q_{j}(z)} g^{j}=1-a_{0} .
$$

i.e.

$$
g^{k-1} g=\frac{1-a_{0}}{a_{k}} \frac{h(z)}{h(z+k c)} e^{-Q_{k}(z)}-\sum_{j=1}^{k-1} \frac{a_{j}}{a_{k}} \frac{h(z+j c)}{h(z+k c)} e^{Q_{j}(z)-Q_{k}(z)} g^{j} .
$$

As $\eta(z)$ is a polynomial, so $\rho_{2}(h) \leq \rho_{2}\left(e^{\eta}\right)=\rho(\eta)=0$, which implies $\rho_{2}(h)=0$. Since $\rho(h)-1<\rho(f)-1=\rho\left(e^{\eta}\right)-1=n-1=\rho(g)$, so by Lemma 3.3, for any $\epsilon>0$, $m\left(r, \frac{h(z+j c)}{h(z+k c)}\right)=o\left(\frac{T(r, h)}{r^{1-\epsilon}}\right)=S(r, g), j=0,1, \ldots, k-1$.

Let $H(z, g)=\sum_{j=0}^{k-1} C_{j} g^{j}$, where $C_{j}=\frac{a_{j}}{a_{k}} \frac{h(z+j c)}{h(z+k c)} e^{Q_{j}(z)-Q_{k}(z)}$ for $j=1,2, \ldots, k-1$ and $C_{0}=\frac{1-a_{0}}{a_{k}} \frac{h(z)}{h(z+k c)} e^{-Q_{k}(z)}$. Thus, (4.5) can be written as $g^{k-1} g=H(z, g)$. Clearly total degree of $H(z, g)$ is at most $k-1$ and $m\left(r, C_{j}\right)=S(r, g)$ for $j=0,1, \ldots k-1$. Hence by Lemma 3.6, $m(r$, $g)=S(r, g)$ that means $T(r, g)=S(r, g)$, a contradiction. Therefore $\operatorname{deg}(\eta(z))=1$. Let us assume that $\eta(z)=\mu z+C$, where $\mu$ and $C$ be two nonzero constants.

Step 4. In this step we deduce a necessary condition and actual form of the function. 
Putting $\eta(z)=\mu z+C$ in $f(z)=h(z) e^{\eta(z)}$, we have $f(z)=A h(z) e^{\mu z}$, where $A=e^{C}$ is a nonzero constant. Now, applying this, (4.4) can be written as

$$
\sum_{j=0}^{k} a_{j} h(z+j c)\left(e^{\mu c}\right)^{j}=h(z) .
$$

i.e.

$$
\sum_{j=0}^{k} a_{j} \frac{h(z+j c)}{h(z)}\left(e^{\mu c}\right)^{j}=1
$$

Entire functions of restricted hyper-order

Since $\rho(h)<\rho(f)=\rho\left(e^{\mu z}\right)=1$, so by Lemma 3.5, there exist $\epsilon$-set $E$, as $z \notin E$ and $z \rightarrow \infty$, such that $\frac{h(z+j c)}{h(z)} \rightarrow 1$. Thereby,

$$
\sum_{j=0}^{k} a_{j} e^{\mu c j}=1
$$

Since $\rho(h)<1$, so by Lemma 3.8, we know that $h(z)$ is a polynomial. Let us assume that $h(z)=c_{k} z^{l}+c_{l-1} z^{l-1}+\cdots+c_{1} z+c_{0}$. Putting it into (4.6) and then comparing coefficients and doing a simple calculation, we have (4.7) and

$$
\begin{gathered}
l \sum_{j=1}^{k} j a_{j} e^{\mu c j}=0, \quad(l-1) \sum_{j=1}^{k} j^{2} a_{j} e^{\mu c j}=0, \\
(l-2) \sum_{j=1}^{k} j^{3} a_{j} e^{\mu c j}=0, \ldots \ldots, \quad(l-(l-1)) \sum_{j=1}^{k} j^{l} a_{j} e^{\mu c j}=0 .
\end{gathered}
$$

Without loss of generality, we assume that all $a_{i}^{\prime} \mathrm{s}$ for $i=1,2, \ldots, k$ are nonzero. Now, the above system of equations can be written as

where $A_{1}=\left(\begin{array}{ccc}1 & 1 & 1 \ldots 1 \\ 1 & 2 & 3 \ldots k \\ 1^{2} & 2^{2} & 3^{2} \ldots k^{2} \\ \ldots & \ldots & \ldots \\ 1^{l} & 2^{l} & 3^{l} \ldots k^{l}\end{array}\right)_{(l+1) \times k} \quad, X=B\left(\begin{array}{c}a_{1} e^{\mu c} \\ a_{2} e^{2 \mu c} \\ a_{3} e^{3 \mu c} \\ \ldots \\ a_{k} e^{k \mu c}\end{array}\right)_{k \times 1}$ and $B=\left(\begin{array}{c}1-a_{0} \\ 0 \\ 0 \\ \ldots \\ 0\end{array}\right)_{(l+1) \times 1}$.

Let $C$ be the corresponding augmented matrix. It is obvious that $\operatorname{rank}\left(A_{1}\right)=\min \{l+1, k\}$. Clearly $\operatorname{rank}(C)=\min \{l+1, k+1\}$.

Suppose $a_{0} \neq 1$. So the nonhomogeneous system (4.8) has unique solution when $l=k-1$, infinitely many solutions when $l<k-1$ and no solutions when $l>k-1$. Hence $\operatorname{deg}(h) \leq(k-1)$.

Next suppose $a_{0}=1$. Then for $k=1, L_{c} f=f$ and (4.7) both implies $a_{1}=0$, which is not possible. So in this case obviously $k \geq 2$. Now, the homogeneous system $A_{1} X=0$ has solutions when $l \leq k-2$. Thus, we have our desired Conclusion 1.

Case 2. Suppose $\bar{N}\left(r, 0 ; e^{\eta}-\frac{a}{h}, e^{\eta}-\frac{b}{q}\right) \neq S(r)$. Let $z_{2}$ is a zero of $e^{\eta}-\frac{a}{h}$ and $e^{\eta}-\frac{b}{q}$. It is obvious that $z_{2}$ is a zero of $\frac{a}{h}-\frac{b}{q}$. If $\frac{a}{h}-\frac{b}{q} \not \equiv 0$, then 


$$
\bar{N}\left(r, 0 ; e^{\eta}-\frac{a}{h}, e^{\eta}-\frac{b}{q}\right) \leq \bar{N}\left(r, \frac{1}{\frac{a}{h}-\frac{b}{q}}\right)=S(r),
$$

which is a contradiction. Therefore

$$
\frac{a}{h}=\frac{b}{q} .
$$

Since $a \not \equiv 0$, therefore $b \not \equiv 0$. Let $z_{3}$ be a zero of $e^{\eta}-\frac{b}{h}$ but $q\left(z_{0}\right) \neq 0$. Since $h e^{\eta}$ and $q e^{\eta}$ share the set $\{a, b\} \mathrm{IM}$, so $z_{3}$ is a zero of $e^{\eta}-\frac{a}{q}$ or $e^{\eta}-\frac{b}{q}$. Let us denote by $\bar{N}\left(r, 0 ; e^{\eta}-\frac{b}{h}, e^{\eta}-\frac{a}{q}\right)$ the reduced counting function of those common zeros of $e^{\eta}-\frac{b}{h}$ and $e^{\eta}-\frac{a}{q}$, which are not zeros of $q$. similarly, we denote by $\bar{N}\left(r, 0 ; e^{\eta}-\frac{b}{h}, e^{\eta}-\frac{b}{q}\right)$ the reduced counting function of those common zeros of $e^{\eta}-\frac{b}{h}$ and $e^{\eta}-\frac{b}{q}$, which are not zeros of $q$. Therefore from (4.3) we have,

$$
\begin{aligned}
T\left(r, e^{\eta}\right) & =\bar{N}\left(r, \frac{1}{e^{\eta}-\frac{b}{h}}\right)+S(r) \\
& =\bar{N}\left(r, 0 ; e^{\eta}-\frac{b}{h}, e^{\eta}-\frac{a}{q}\right)+\bar{N}\left(r, 0 ; e^{\eta}-\frac{b}{h}, e^{\eta}-\frac{b}{q}\right)+S(r),
\end{aligned}
$$

which shows that either $\bar{N}\left(r, 0 ; e^{\eta}-\frac{b}{h}, e^{\eta}-\frac{a}{q}\right) \neq S(r)$ or $\bar{N}\left(r, 0 ; e^{\eta}-\frac{b}{h}, e^{\eta}-\frac{b}{q}\right) \neq S(r)$. Otherwise $T\left(r, e^{\eta}\right)=S(r)$, which is not possible in view of (4.1). Now, we consider two subcases:

Subase 2.1. Suppose $\bar{N}\left(r, 0 ; e^{\eta}-\frac{b}{h}, e^{\eta}-\frac{a}{q}\right) \neq S(r)$. Then proceeding in a similar manner as used in starting portion of Case 2 , we have $\frac{b}{h}=\frac{a}{q}$. In view of (4.9) we get, $a^{2}=b^{2}$. As $a \not \equiv b$, so obviously $b=-a$. Therefore we must have $q=-h$ that implies $L_{c} f=-f$. Further following the same steps as done in Case 1, we can have the form of the function as $f(z)=A h(z) e^{\mu z}$ satisfying

$$
\sum_{j=0}^{k} a_{j} e^{\mu c j}=-1
$$

Next adopting the similar calculations as done in Step 4 , for $a_{0} \neq-1$, we have $\operatorname{deg}(h) \leq(k-1)$ and for $a_{0}=-1$, we have $k \geq 2$ and $\operatorname{deg}(h) \leq(k-2)$. Thus, we have corresponding desired conclusion (2).

Subcase 2.2. Suppose $\bar{N}\left(r, 0 ; e^{\eta}-\frac{b}{h}, e^{\eta}-\frac{b}{q}\right) \neq S(r)$, which is similar to the Case 1 and so, we get the desired result.

Hence the proof is completed.

Proof of Corollary 2.1. To prove this corollary, it is sufficient to prove that $\operatorname{deg}(h)=0$, where $h(z)=c_{k} k^{l}+c_{l-1} z^{l-1}+\cdots+c_{1} z+c_{0}(l \leq k-1), c_{l} \neq 0$. We know for the operator $\Delta_{c}^{k} f$, $a_{j}=(-1)^{k-j}\left(\begin{array}{l}k \\ j\end{array}\right)$, where $j=0,1, \ldots, k$.

From conclusion 1. of Theorem 2.1 we have, $\Delta_{c}^{k} f=f$ and $f$ takes the form $f=A h(z) e^{\mu z}$, where $A$ is a nonzero constant, $h(z)$ is a polynomial and $\mu$ is a nonzero constant satisfying $\sum_{j=0}^{k}(-1)^{k-j}\left(\begin{array}{l}k \\ j\end{array}\right) e^{\mu c j}=1$, that is, $\left(e^{\mu c}-1\right)^{k}=1$. Now putting $f=A\left(c_{l} z^{l}+c_{l-1} z^{l-1}\right.$ $\left.+\cdots+c_{1} z+c_{0}\right) e^{\mu z}$ into $\Delta_{c}^{k} f=f$ and then comparing coefficient of $z^{k-1}$, we have 


$$
\begin{aligned}
& \sum_{j=1}^{k}\left(l c_{l} j c+c_{l-1}\right)(-1)^{k-j}\left(\begin{array}{c}
k \\
j
\end{array}\right) e^{\mu c j}=c_{l-1} \\
& \Rightarrow l c_{l} c \sum_{j=1}^{k} j(-1)^{k-j}\left(\begin{array}{c}
k \\
j
\end{array}\right) e^{\mu c j}+c_{l-1}\left(e^{\mu c}-1\right)^{k}=c_{l-1} .
\end{aligned}
$$

Entire functions of restricted hyper-order

As $c \neq 0, c_{l} \neq 0$ and $\left(e^{\mu c}-1\right)^{k}=1$ and also we know $j\left(\begin{array}{l}k \\ j\end{array}\right)=k\left(\begin{array}{l}k-1 \\ j-1\end{array}\right)$, so we get

$$
\begin{aligned}
& l \sum_{j=1}^{k}(-1)^{k-j} k\left(\begin{array}{c}
k-1 \\
j-1
\end{array}\right) e^{\mu c j}=0 \\
& \Rightarrow l \sum_{j=0}^{k-1}(-1)^{(k-1)-j}\left(\begin{array}{c}
k-1 \\
j
\end{array}\right) e^{\mu c j}=0 \Rightarrow l\left(e^{\mu c}-1\right)^{k-1}=0 .
\end{aligned}
$$

Clearly in view of $\left(e^{\mu c}-1\right)^{k}=1, l$ must be 0 .

From conclusion 2. of Theorem 2.1 we have, $b=-a, \Delta_{c}^{k} f=-f$ and $f$ takes the form $f=A h(z) e^{\mu z}$, where $A$ is a nonzero constant, $h(z)$ is a polynomial and $\mu$ is a nonzero constant satisfying $\sum_{j=0}^{k}(-1)^{k-j}\left(\begin{array}{l}k \\ j\end{array}\right) e^{\mu c j}=-1$, that is, $\left(e^{\mu c}-1\right)^{k}=-1$. Here obviously $k \geq 2$. Proceeding in a similar manner as in above, again we can have (4.11) and in view of $\left(e^{\mu c}-1\right)^{k}=-1$, we can conclude $l=0$. So in both cases $l=0$ that means $\operatorname{deg}(h)=0$. Hence the corollary is proved.

\section{Observation and an open question}

As we know $\lambda(f) \leq \rho(f)$ and since throughout the paper we have dealt with the case $\lambda(f)<\rho(f)$, it will be interesting to inspect whether the same conclusions hold for the case $\lambda(f)=\rho(f)$. In the next two examples we point out the fact that when $\lambda(f)=\rho(f)$, the conclusion of Theorem 2.1 ceases to hold.

Example 5.1. Let $f=e^{z}\left(e^{2 z}+1\right)$. Choose $c=\pi i$ and for even integer $k, a_{k}+\cdots+a_{2}+a_{0}=0$ and $a_{k-1}+\cdots+a_{3}+a_{1}=1$. Then $\lambda(f)=\rho(f)=1$ and $L_{c} f=-e^{z}\left(e^{2 z}+1\right)$. Clearly $L_{c} f$ and $f$ share the set $\{a,-a\} C M$, where $a$ is an entire function such that $\rho(a)<\rho(f)$. Though $L_{c} f=-f$, the form of $f$ does not satisfy the conclusion of Theorem 2.1.

Example 5.2. Let $f=-e^{z}+3$ and $\left(e^{c}-1\right)^{2}=-1$. Then $\Delta_{c}^{2} f=e^{z}$ and $\lambda(f)=\rho(f)=1$. Clearly $\Delta_{c}^{2} f$ and $f$ share the set $\{1,2\}$ CM but $\Delta_{c}^{2} f \neq \pm f$.

In view of the above two examples, we can conclude that in Theorem $2.1, \lambda(f)<\rho(f)$ is sharp, but the conclusion of the same theorem under the case $\lambda(f)=\rho(f)$ is still an enigma. So we place it as an open question:

Question 5.1. Under the hypothesis $\lambda(f)=\rho(f)$, what will be the answer of the Question 1.1 concerning $\Delta_{c}^{k}$ f or even $L_{c} f$ ?

\section{References}

1. Hayman, WK. Meromorphic functions. Oxford: The Clarendon Press; 1964.

2. Li, P, Yang, CC. Value sharing of an entire function and its derivatives. J Math Soc Japan. 1999; 51(4): 781-99. 
3. Li, P. Value sharing and differential equations. J Math Anal Appl. 2005; 310: 412-23.

4. Liu, K. Meromorphic functions sharing a set with applications to difference equations. J Math Anal Appl. 2009; 359: 384-93.

5. Li, XM. Entire functions sharing a finite set with their difference operators. Comput Meth Func Theor. 2012; 12(1): 307-28.

6. Qi, J, Wang, Y, Gu, Y. A note on entire functions sharing a finite set with their difference operators. Adv Differ Equ. 2019; 114: 1-7.

7. Bergweiler, W, Langley, JK. Zeros of differences of meromorphic function. Math Proc Camb Philos Soc. 2007; 142: 133-47.

8. Chiang, YM, Feng, SJ. On the Nevanlinna Characteristic $f(z+\eta)$ and difference equations in complex plane, Ramanujan J. 2008; 16: 105-29.

9. Halburd, RG, Korhonen, RJ, Tohge, K. Holomorphic curves with shift invariant hyperplane preimages. Trans Am Math Soc. 2014; 366: 4267-98.

10. Laine, I. Nevanlinna theory and complex differential equations, studies in math. Berlin: de Guryter. 1993; 15.

11. $\mathrm{Lu}, \mathrm{F}, \mathrm{Lu}, \mathrm{W}, \mathrm{Li}, \mathrm{C}, \mathrm{Xu}, \mathrm{J}$. Growth and uniqueness related to complex differential and difference equations. Results Math. 2019; 74. Art. 30: 1-18.

12. Yang, CC, Yi, HX. Uniqueness theory of meromorphic functions. Dordrecht: Kluwer Academic Publishers; 2003.

\section{Corresponding author}

Arpita Roy can be contacted at: arpita140793@gmail.com

For instructions on how to order reprints of this article, please visit our website: 